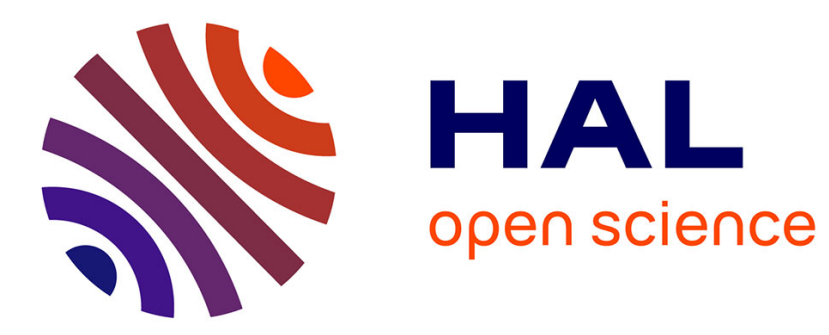

\title{
Les systèmes de tons en Asie orientale: typologie, schémas évolutifs et modélisation
}

\author{
Alexis Michaud
}

\section{To cite this version:}

Alexis Michaud. Les systèmes de tons en Asie orientale: typologie, schémas évolutifs et modélisation.

Faits de langues, 2011, 37, pp.247-261. halshs-00436458v2

\section{HAL Id: halshs-00436458 \\ https://shs.hal.science/halshs-00436458v2}

Submitted on 12 Apr 2013

HAL is a multi-disciplinary open access archive for the deposit and dissemination of scientific research documents, whether they are published or not. The documents may come from teaching and research institutions in France or abroad, or from public or private research centers.
L'archive ouverte pluridisciplinaire HAL, est destinée au dépôt et à la diffusion de documents scientifiques de niveau recherche, publiés ou non, émanant des établissements d'enseignement et de recherche français ou étrangers, des laboratoires publics ou privés. 


\title{
Les systèmes de tons en Asie orientale : typologie, schémas évolutifs et modélisation *
}

\begin{abstract}
Alexis Michaud
Résumé: La présente contribution récapitule certaines avancées majeures réalisées dans l'étude des systèmes tonals d'Asie orientale, et discute leurs implications pour la typologie tonale et la modélisation phonologique. Les travaux phonologiques et typologiques accordent une place relativement limitée aux langues dans lesquelles les tons ne sont pas définis simplement en termes de hauteur, et ne se prêtent pas à une décomposition en niveaux tonals (par exemple le tamang et le vietnamien). Ces langues manquent assurément d'attrait au plan morpho-phonologique, étant dénuées d'alternances tonales. Elles n'en présentent pas moins une complexité bien réelle, qui doit être prise en considération dans la modélisation phonologique et les généralisations typologiques. Le panorama présenté ici ramène à une distinction proposée il y a plus d'un demi-siècle (Pike 1948) entre deux types d'organisation tonale : d'une part les systèmes de tons ponctuels*, d'autre part les systèmes de tons complexes*. Pour approfondir cette distinction synchronique, une attention particulière est accordée à l'évolution des systèmes tonals : les faits d'évolution sont en effet d'excellents révélateurs de la nature des systèmes phonologiques.

Abstract: This contribution recapitulates major findings about East Asian languages to discuss general issues of tonal typology, diachronic evolution and phonological modeling. Relatively little attention has been paid to Far Eastern languages in which linguistic tone cannot simply be equated with pitch, or be

\footnotetext{
* Merci à Guillaume Jacques, Martine Mazaudon, Boyd Michailovsky, Laurent Sagart et Jacqueline Vaissière pour leurs précieux commentaires. Mes remerciements tout particuliers à Michel Ferlus pour les informations au sujet de systèmes tonals tai-kadai et austroasiatiques. Cet article a également bénéficié d'une réflexion commune avec Nick Clements et Cédric Patin au sujet de l'analyse des tons en traits (cf. Clements et al. forthcoming). Il va de soi que je suis seul responsable des interprétations proposées ici.
} 
decomposed into tonal levels, such as Tamang or Vietnamese. These languages are admittedly somewhat unattractive for phonologists, since they lack tonal alternations. However, they clearly need to be taken into account in phonological modeling and typological generalizations - and they present highly interesting complexities of their own. The conclusion which emerges in light of a synchronic and diachronic survey is very close to a point of view expressed more than half a century ago (Pike 1948), namely that there exists a major typological divide between two types of tonal organization: (i) level-tone systems and (ii) complex-tone systems. With a view to drawing all the implications of this divide, special attention is devoted to the evolution of tonal systems: diachronic facts provide precious insights into the nature of phonological systems.

\section{INTRODUCTION}

Depuis le début du $\mathrm{XX}^{\mathrm{e}}$ siècle, des progrès considérables ont été réalisés dans l'étude des systèmes tonals des langues de l'Asie orientale. Les progrès les plus clairs concernent la linguistique historique : l'origine diachronique des tons d'un grand nombre de langues est désormais bien établie. Au plan de la typologie tonale et de la modélisation phonologique des tons* ${ }^{*}$, en revanche, des incompréhensions demeurent entre spécialistes de domaines géographiques différents. Certaines conclusions des chercheurs orientalistes continuent de susciter étonnement et incrédulité auprès de chercheurs familiers du domaine subsaharien ; inversement, l'application systématique aux langues asiatiques de modèles développés à l'origine pour des langues africaines (dans lesquelles tous les tons sont réductibles à des tons ponctuels*) soulève des objections de la part de spécialistes de ces langues.

Le présent article situe les faits dans une perspective typologique. Les systèmes tonals d'Asie orientale sont d'une grande diversité, notamment en termes de domaine tonal (syllabe ou mot) et de corrélats phonétiques des tons. Pour autant, l'examen des résultats de recherche accumulés amène à reconnaître deux types fondamentaux de systèmes tonals non réductibles l'un à l'autre : tons ponctuels*, décomposables en niveaux de hauteur, et tons complexes*, non réductibles à des niveaux. Sur ce point, les résultats les plus récents nous ramènent à une distinction qui avait déjà été proposée il y a plus d'un demi-siècle (Pike 1948).

\footnotetext{
${ }^{1}$ Les astérisques renvoient au court Glossaire proposé en fin d'article.
} 


\section{TYPOLOGIE ET MODELISATION}

\subsection{Une distinction fondamentale : tons ponctuels et tons complexes}

Il est indispensable de situer la modélisation des systèmes tonals dans un cadre typologique. Une typologie tonale est proposée par Pike 1948, distinguant deux types de systèmes. L'un repose sur des tons simples, définis par un niveau de hauteur: les tons ponctuels*. L'autre repose sur des tons complexes* (que Pike appelait tons modulés; en anglais contour tones). Cette distinction typologique se recoupe partiellement avec la distribution géographique des systèmes tonals : les systèmes de tons ponctuels dominent dans le domaine subsaharien (voir en particulier Clements et Goldsmith 1984), et les systèmes de tons complexes en Asie orientale. Le caractère complexe de systèmes tonals d'Asie orientale comme ceux du vietnamien ou du chinois a été observé de longue date par les spécialistes de ces langues; ainsi des tons du chinois: "Chinese tones are complex suprasegmental events distinguishing (otherwise) homophonous syllables » (Sagart 1999:92). L'évidence des faits observés s'impose avec une telle force que les spécialistes ont naturellement tendance à prêter le statut d'universaux des systèmes tonals aux caractéristiques qu'ils observent de façon répétée dans les langues qu'ils examinent, et à considérer avec suspicion les travaux qui (partant de langues différentes) parviennent à des conclusions si différentes des leurs. Les auteurs qui étudient des systèmes de tons complexes peuvent douter de l'existence même de systèmes de tons définis simplement par des niveaux de hauteur (les tons ponctuels*). On trouve ainsi chez Eugénie Henderson une mise en garde : «... 'tone' is seldom, if ever, a matter of pitch alone » (Henderson 1965:404) ; le if ever revient à jeter le doute sur la validité des descriptions de systèmes tonals qui ne prendraient pas en compte les types de phonation* et autres corrélats phonétiques potentiels des tons. Pareillement, Philip Rose a publié un travail intitulé « De la non-équivalence entre fréquence fondamentale et ton linguistique » (Rose 1989a). A l'inverse, la phonologie générative a rapidement fait siens les modèles tonals (dits 《 autosegmentaux ») développés pour les tons ponctuels* des langues subsahariennes, qui se sont trouvés proposés au statut de modèle universel des tons; les tenants de la phonologie générative ont par conséquent un a priori défavorable contre la reconnaissance de l'existence d'un type de système différent, fondé sur des tons complexes*.

Le fait que peu de chercheurs aient eu l'occasion d'étudier l'un et l'autre type de système tonal a certainement beaucoup contribué à retarder les progrès de la 
typologie tonale. Dans l'élaboration de travaux d'orientation typologique, il est en principe nécessaire de tenir compte de toutes les données existantes. En pratique, il n'est pas toujours facile de trouver un équilibre entre les deux extrêmes qui consisteraient, soit à ne prendre en compte que les langues dont on est familier, ce qui limite d'emblée la portée du travail, soit à prendre en compte une grande masse de données qui ne sont connues que de deuxième main, avec les risques d'erreurs que cela comporte. Beaucoup d'auteurs font le choix - tout à fait compréhensible - de se fonder essentiellement sur les langues qu'ils connaissent. Ainsi d'Eugénie Henderson : «My preference, derived both from professional training and experience, would be to present only material of which I have first-hand personal knowledge, since, though this may be fallible, one may at least suppose the same bias to run through the whole of it » (Henderson 1965:403). Pareillement, les propositions typologiques formulées par Larry Hyman (Hyman 2006, 2007a) reposent sur sa compréhension en profondeur des systèmes de tons ponctuels* de langues africaines (voir Hyman 2001, et de nombreux autres articles) ainsi qu'asiatiques (Hyman et VanBik 2002 ; Hyman 2007b), tandis que les systèmes de tons complexes*, qui n'ont pas eu une place comparable dans son expérience de chercheur, sont quasi absents de ses propositions typologiques.

J'ai pour ma part la grande chance d'être familier à la fois de systèmes de tons ponctuels* - ceux d'une langue sino-tibétaine, le naxi, et de langues proches parentes, na et lazé, dont les unités tonales de base sont de simples niveaux: $\mathrm{H}($ aut), $\mathrm{M}($ oyen) et $\mathrm{B}(\mathrm{as})$ - et de systèmes de tons complexes* (en particulier celui du vietnamien). Cette expérience diversifiée contribuera, je l'espère, à lever les soupçons de parti pris en faveur de telle ou telle approche théorique. L'antagonisme entre modèles « africanistes » (modélisation " autosegmentale », uniquement en tons ponctuels*) et «orientalistes» (description des tons complexes* comme unités distinctives non décomposables) disparait en réalité dès lors que l'on reconnaît que leur domaine d'application typologique est différent. Les modèles autosegmentaux sont adaptés aux systèmes de tons ponctuels*, non aux tons complexes*.

\subsection{Nouveaux arguments soutenant la dichotomie classique entre tons ponctuels et tons complexes : l'exemple du chinois mandarin}

Les dialectes chinois (ou «langues sinitiques») constituent un domaine linguistique dans lequel les tons sont traditionnellement considérés comme des tons complexes*. Les travaux fondateurs de Chao Yuen-ren $(1929,1933)$, qui a collaboré avec Kenneth Pike, ont été poursuivis par divers auteurs; la 
description du système tonal du dialecte de Zhenhai par Rose 1990 illustre le cas des systèmes dont les tons sont définis non seulement par un contour mélodique mais aussi par un certain type de phonation* (voir également Rose 1982, 1989b).

Ces conclusions ont été remises en cause par des auteurs travaillant dans le cadre de la phonologie générative, qui ont avancé diverses propositions d'analyses théoriques décomposant ces tons en "atomes tonals » plus petits (de simples tons ponctuels*). Avec le recul, ces modèles s'avèrent peu éclairants : ils ne parviennent pas à expliquer les phénomènes de sandhi tonal de ces langues, et sont réduits à les modéliser au moyen de règles ad hoc, souvent d'une grande complexité (une critique de l'analyse du cantonais en termes autosegmentaux est proposée par Clements et al. forthcoming).

Un point positif pour la typologie tonale est que les différences typologiques entre systèmes tonals semblent s'imposer à nouveau à l'attention des praticiens de la phonologie générative. Certains auteurs reconnaissent désormais l'impossibilité de modéliser de la même façon les ensembles typologiques traditionnellement décrits comme tons ponctuels (niveaux tonals), d'une part, comme tons complexes (ou contours non analysables), d'autre part. En témoigne le constat suivant :

" [In African languages] contour tones were clearly shown to be composed of a sequence of level tones. The phenomena that gave rise to the analyses of contour tones as such are not found in Chinese languages; however, in the interest of theoretical parsimony, the same analysis in which contour tones are treated as a sequence of level tones was carried over to Chinese tonal systems, in the absence of evidence to the contrary. However, we have seen enough evidence to doubt that a sequence of level tones is an appropriate representation for the contour tones under discussion and that, in fact, contour tones in Chinese tonal languages should be seen as unitary. » (Barrie 2007:345)

Les divers modèles proposés dans le cadre de la phonologie générative pour représenter ces deux types d'organisation des systèmes tonals sont très différents entre eux, voire franchement contradictoires (voir par exemple Yip 1989 et Bao 1999:200-206); cette situation, qui tranche avec les progrès remarquables réalisés dans l'analyse des tons ponctuels, met indirectement en lumière les différences de structure qui séparent ces types d'organisation tonale.

La modélisation phonologique des tons complexes* soulève des questions épineuses, à commencer par celle des critères utilisés pour déterminer si un système tonal repose sur des tons ponctuels ou sur des tons complexes. La présence d'alternances tonales (morphologiques ou phonologiques) peut permettre de démontrer de façon décisive que les tons en question sont des tons ponctuels. Les exemples dans le domaine subsaharien sont très abondants ; dans 
le domaine asiatique, les exemples les plus probants comprennent le hakha lai (Hyman et VanBik 2002), le pumi/prinmi (Ding 2006 ; Jacques, en préparation), le shixing (Chirkova et Michaud 2009), et le naxi. Dans cette dernière langue, un ton $\mathrm{H}$ (aut) d'origine morphosyntaxique peut s'associer à une syllabe précédente ayant un ton lexical $\mathrm{B}$ (as) ou $\mathrm{M}$ (oyen), avec pour effet la création de séquences tonales BH et MH, respectivement (Michaud 2006 ; Michaud et He Xueguang 2007). Une synthèse au sujet des systèmes de tons ponctuels* en Extrême-Orient est proposé par Evans 2008.

En revanche, il est beaucoup plus difficile de démontrer de façon convaincante le caractère non décomposable de tons complexes, puisque l'échec de la tentative d'analyse pourrait en théorie être dû à une analyse insuffisamment poussée, plutôt qu'à un caractère réellement indécomposable des tons en question. En dépit de ce problème de méthode, il existe des arguments pertinents pour aborder cette question, tels que les lapsus. Si un ton consiste en une séquence de niveaux simples (par exemple : un ton $\mathrm{BH}$, formé de la succession d'un niveau Bas et d'un niveau Haut), on s'attend à observer certains lapsus dans lesquels seule une de ces deux unités serait omise (réalisation comme un simple $\mathrm{H}$, ou un simple B). Or des observations réalisées au sujet du chinois mandarin (Wan et Jaeger 1998) suggèrent au contraire que dans cette langue, lors des « lapsus tonals ", l'un des quatre tons du système se substitue à un autre, dans son entier. L'interprétation retenue par les auteurs de l'étude est qu'un ton se substitue à un autre de façon globale, ce qui porte à reconnaître l'existence de tons complexes*, dont l'organisation phonologique diffère de celle des tons ponctuels* : «phonological theories which require that all contour tones in every language must be represented as a sequence of level tones underlyingly may be missing an insight into the possible underlying differences among tone languages» (Wan et Jaeger 1998:458). Cette prudente conclusion rejoint le constat formulé par plusieurs praticiens de l'analyse tonale dans le domaine extrême-oriental, par exemple Jackson Sun: «tone-spreading or melodymapping analyses, useful as they are for languages like New Shanghai, should be utilized only where they are really called for with languages like Tibetan » (Sun 1997:516).

La reconnaissance de deux types d'organisation tonale non réductibles l'un à l'autre soulève la question de l'origine de cette différence, suivant en cela la ligne directrice suggérée par les éditeurs du présent volume, qui soulignent le fait que la parole est inscrite dans un processus évolutif. La section 2 vise à réunir et synthétiser connaissances et hypothèses à ce sujet. 


\section{SCHEMAS EVOLUTIFS : ORIGINE DES TONS COMPLEXES*; REGISTROGENESE ET} TONOGENESE

La présente section résume brièvement des acquis importants au sujet des schémas d'évolution aboutissant à la création de tons, puis développe une hypothèse au sujet du lien entre les deux types synchroniques décrits en section 1 (tons ponctuels* et tons complexes*), d'une part, et deux types d'évolution diachronique, d'autre part.

\subsection{Tonogenèse et registrogenèse}

La tonogenèse peut résulter de la perte d'oppositions entre séries de consonnes initiales de syllabe, ou de la perte de consonnes finales. Le changement se fait par un mécanisme de compensation (appelé transphonologisation): les oppositions lexicales sont conservées par le biais d'un nouveau type d'opposition.

Si la modélisation phonologique des tons complexes* est plus problématique que celle des tons ponctuels, au plan diachronique, en revanche, l'origine des systèmes de tons complexes du domaine asiatique est désormais bien connue. Reprenons l'exemple du vietnamien. On doit la première analyse détaillée de l'origine des tons du vietnamien à Haudricourt 1954, 1961. Le tableau 1 récapitule l'évolution d'un état où la langue n'avait aucun ton jusqu'au système actuel. L'évolution se fait par transphonologisation d'oppositions consonantiques en oppositions tonales.

Tableau 1. Les tons du vietnamien en perspective diachronique.

Tableau 1a: les rimes du protoviet-muong tardif: syllabes ouvertes sans glottalisation; constriction glottale finale; $/ \mathrm{h} /$ final ; /p/, /t/ ou $/ \mathrm{k} /$ final. D'après Ferlus 2001:2.

\begin{tabular}{|l|l|l|}
\hline ta & ta $^{\text {? }}$ & tah \\
\hline da & da? $^{\text {? }}$ & dah \\
\hline
\end{tabular}

tap, tat, tak

dap, dat, dak
Tableau $1 \mathrm{~b}$ : première étape de la tonogenèse en vietnamien: trois tons ; pas d'oppositions tonales sur les syllabes à occlusive finale.

\begin{tabular}{|l|l|l|l|l|l|}
\hline ta & ton $\mathrm{A}$ & ta & ton $\mathrm{B}$ & ta & ton C \\
\cline { 3 - 4 } & & $\mathrm{da}$ & & $\mathrm{da}$ & \\
\hline & & $\begin{array}{l}\text { catégorie D } \\
\text { (sap, tat, tak }\end{array}$ \\
\hline dap, dat, dak & (sans ton) \\
\hline
\end{tabular}


Tableau 1c : le système tonal du vietnamien contemporain (parler de Hanoi) : catégories tonales en notation étymologique, et nom par lequel ils sont désignés dans la tradition orthographique. Les tons A1 à C2 n'apparaissent que sur les syllabes ouvertes ou à nasale finale, les tons D1 et D2 sur les syllabes à occlusive finale.

\begin{tabular}{|l|l|l|l|l|l|}
\hline ta & $\begin{array}{l}\text { ton } \mathrm{A} 1 \\
\text { (ngang) }\end{array}$ & ta & $\begin{array}{l}\text { ton B1 } \\
\text { (sắc) }\end{array}$ & ta & $\begin{array}{l}\text { ton C1 } \\
\text { (hỏi) }\end{array}$ \\
\hline ta & $\begin{array}{l}\text { ton } \mathrm{A} 2 \\
\text { (huyền) }\end{array}$ & ta & $\begin{array}{l}\text { ton B2 } \\
\text { (nặng) }\end{array}$ & ta & $\begin{array}{l}\text { ton C2 } \\
\text { (ngã) }\end{array}$ \\
\hline
\end{tabular}

\begin{tabular}{|l|l|}
\hline tap, tat, tak & tone D1 (sắc) \\
\hline tap, tat, tak & tone D2 (nặng) \\
\hline
\end{tabular}

L'établissement de ce schéma historique pose la question phonétique des états intermédiaires, du processus même par lequel ont lieu ces transphonologisations. Pulleyblank 1978 a été le premier à suggérer une étape de types phonatoires dans la bipartition en chinois : «...the split into upper and lower registers was conditioned primarily by the voiced aspiration, giving rise to breathy vowels, rather than simple voice» (Pulleyblank 1978:173). Les travaux fondateurs concernant les types phonatoires - par exemple l'opposition entre des syllabes réalisées en voix modale et en voix soufflée/murmurée dans la langue mon sont en fait contemporains de la découverte de l'origine des tons du vietnamien par Haudricourt. L'étude de la langue khmer par Henderson 1952 avait déjà permis de comprendre comment une ancienne opposition de voisement sur les consonnes initiales avait pu donner une opposition de registre*, puis une opposition de timbre vocalique. L'article de Ferlus 1979 au sujet des liens entre mutations consonantiques, types de phonation, modifications de timbre vocalique et tons dans les langues mon-khmer demeure aujourd'hui la meilleure synthèse sur ce sujet. On ne saurait trop insister sur l'importance de cet article, qui expose les mécanismes par lesquels une opposition de consonnes initiales se transphonologise, contribuant à éclairer les liens diachroniques entre consonnes, registres* et tons* (voir également Ferlus 2009).

Pour résumer, les deux principales sources diachroniques de tons lexicaux résident dans les consonnes finales et les consonnes initiales - même si certaines 
langues soumises à l'influence de langues de prestige voisines possédant des tons peuvent transphonologiser en tons d'autres types d'oppositions : ainsi de la langue hu, d'après Svantesson 2001. Le paragraphe qui suit examine l'hypothèse selon laquelle l'origine diachronique des tons déterminerait leur nature phonologique.

\subsection{Les tons complexes, résultat d'un télescopage entre deux stades de la tonogenèse?}

Une hypothèse concernant les liens entre origine diachronique et nature synchronique est proposée par Michel Ferlus (communication personnelle) : si la tonogenèse par les consonnes finales en est encore à un stade qui comporte des caractéristiques phonatoires (registrales) au moment où s'engage la tonogenèse par les initiales, les traces de caractéristiques phonatoires ont toutes chances d'être conservées dans le système tonal après bipartition, comme c'est le cas en vietnamien (où deux des tons comportent une glottalisation). Ces traces peuvent disparaître ensuite, comme c'est le cas en vietnamien du sud (voir l'étude détaillée par Brunelle 2009 des indices perceptifs utilisés par des locuteurs du nord et du sud). Une conclusion qui paraît ressortir de ces faits est que la présence ou l'absence de caractéristiques de phonation (glottalisation ou voix soufflée, par exemple) ne permet pas de fonder une dichotomie typologique. De fait, pour certaines langues (par exemple dans le domaine des dialectes chinois), il est difficile de savoir dans quelle mesure les caractéristiques de phonation occasionnellement présentes pour certains tons font partie de la définition phonologique de ces tons.

\subsection{Evolution diachronique des tons ponctuels* et des tons complexes*. Apport d'une approche panchronique}

La phonologie panchronique (Haudricourt 1969, Hagège et Haudricourt 1978, Mazaudon et Michailovsky 2007) vise à décrire les potentialités évolutives latentes dans un état donné d'un système linguistique. Une approche de la typologie tonale informée par des considérations panchroniques pourrait permettre de mieux appréhender les deux modes d'organisation des systèmes tonals distingués ici : tons ponctuels* et tons complexes*. Les systèmes de tons complexes connaissent une évolution phonétique graduelle, la réalisation phonétique des tons évoluant sans que l'on puisse aisément y distinguer des étapes bien différenciées (confusions tonales pures et simples mises à part). En revanche, la diachronie des systèmes de tons ponctuels est jalonnée de 
changements catégoriels : dans des circonstances données, des différences non contrastives dans la réalisation du ton (variation allotonique conditionnée) sont réinterprétées comme des différences entre catégories tonales, ce qui modifie le système phonologique. Par exemple, il est courant d'observer une réalisation rehaussée d'un ton $\mathrm{H}$ (aut) devant un ton $\mathrm{B}(\mathrm{as})$; pour prendre un exemple gulmancema (tableau 2), la syllabe ${ }^{H} \mathrm{H} k a n /$ sera réalisée phonétiquement plus haute dans la séquence $/{ }^{\mathrm{LM}_{\mathrm{O}}}{ }^{\mathrm{H}} \mathrm{kan}{ }^{\mathrm{L}} \mathrm{di} /$ que dans $/{ }^{\mathrm{LM}} \mathrm{O}{ }^{\mathrm{H}} \mathrm{kan}{ }^{\mathrm{H}} \mathrm{di} / . \mathrm{Ce}$ phénomène phonétique n'affecte pas la nature phonologique des tons; en revanche, en moba, langue proche parente du gulmancema, la variante ultrahaute $\mathrm{du}$ ton $\mathrm{H}$ a acquis valeur contrastive lors de la perte des voyelles finales de mot, ce qui aboutit à l'apparition d'une nouvelle unité tonale dans le système : un ton ultra-haut.

Tableau 2. Données comparatives révélant l'origine du ton ultra-haut du moba. Données et analyse tirées de Rialland 2001:317.

\begin{tabular}{|l|l|l|}
\hline sens & Gulmancema & Moba \\
\hline il a marché sur & ${ }^{\mathrm{LM}} \mathrm{O}{ }^{\mathrm{H}}$ kan ${ }^{\mathrm{L}}$ di ( o kándì ) & ${ }^{{ }^{\mathrm{LM}} \mathrm{u}}{ }^{\mathrm{XH}}$ kant ( ù kấnt ) \\
\hline il marche sur & ${ }^{\mathrm{LM}} \mathrm{O}{ }^{\mathrm{H}}$ kan ${ }^{\mathrm{H}}$ di ( o kándí ) & ${ }^{{ }^{\mathrm{LM}} \mathbf{u}}{ }^{\mathrm{H}}$ kant ( ù kánt) \\
\hline
\end{tabular}

Ceci est un exemple classique de transphonologisation (tranfert d'opposition pertinente), du ton de la voyelle finale vers le ton précédent. La variation allophonique prélude au changement ; le changement lui-même (modification du système) résulte de la perte des voyelles finales.

On ne s'attend pas, dans le cas d'un système tonal qui repose sur une opposition entre niveaux tonals, à ce que les termes de cette opposition en viennent insensiblement à changer de nature, par exemple un ton $\mathrm{H}$ devenant descendant (devenant HM, puis HL). Cela tient à la nature même des tons ponctuels, définis simplement par une valeur sur une échelle monodimensionnelle. A l'opposé, les tons complexes* de langues comme le tamang ou le vietnamien connaissent des changements graduels, sous l'action continue de facteurs phonétiques. A la différence du changement que révèle la comparaison du gulmancema et du moba (voir ci-dessus), l'évolution des contours tonals non décomposables se déroule sans modification du nombre d'unités du système (sauf dans les cas de confusion entre deux tons ; voir par exemple l'étude détaillée des confusions tonales dans les langues Viet-Muong par Ferlus 1998). Les tons complexes sont définis par un contour (auquel s'ajoutent, dans certaines langues, d'autres caractéristiques, notamment de type phonatoire). La caractéristique essentielle de ce contour est d'être distinct de tous les autres; cela laisse une latitude de variation dans la réalisation 
phonétique du ton (son point de départ, son point terminal, le détail de son inflexion...), variation qui a pour effet une évolution diachronique graduelle des tons. L'exemple du tamang et des langues apparentées révèle des évolutions phonétiques, diverses étapes évolutives se trouvant reflétées par la diversité spatiale des dialectes. Le système tonal du tamang de Risiangku illustre une des premières étapes de l'évolution phonétique graduelle des tons complexes : les quatre tons de cette langue, rompant leurs derniers liens avec la corrélation de voisement où réside leur origine diachronique, deviennent libre de s'éloigner de la plage de hauteur qui était la leur à l'origine (tons relativement bas après les anciennes voisées, relativement hauts après les anciennes sourdes). Dans des langues proches parentes, cette évolution est plus avancée : en marphali et dans le dialecte tamang de Taglung, le ton 4 (anciennement de série basse) est désormais devenu phonétiquement haut, de même que le ton 3 en manangke (Mazaudon 1978, 2005, Hildebrandt 2003). «Une fois constitué, le système tonal évolue sans souci de sa valeur ancienne étymologique. » (Haudricourt 1961:165.)

Ces arguments, tirés de la dialectologie, se trouvent confirmés par les études phonétiques, dans le cas des langues pour lesquelles une certaine profondeur de champ historique existe dans les études phonétiques. La généralisation selon laquelle les tons complexes* connaissent une évolution diachronique graduelle se trouve vérifiée dans le cas du thai de Bangkok, qui a été étudié expérimentalement à diverses dates depuis un siècle. Pour prendre un exemple, le ton 4, qui en 1908 était le plus haut du système, et comprenait une descente finale, est maintenant devenu montant (voir la figure 2 de Pittayaporn 2007, qui reproduit les tracés des diverses études menées au cours du $\mathrm{XX}^{\mathrm{e}}$ siècle). Le nombre de tons (unités contrastives) demeure inchangé; leur évolution phonétique est graduelle, l'évolution d'un ton ayant des répercussions sur celle de tel autre ton avec lequel une confusion menace - processus où l'on reconnaît le jeu bien connu de forces antagonistes, tendance à la simplification d'une part, pression pour le maintien des opposition distinctives d'autre part.

La diachronie apparaît dès lors comme un domaine à prendre en compte dans la modélisation phonologique. Sur le papier, il est fort aisé d'avancer une analyse synchronique de n'importe quel système tonal en termes de tons ponctuels* : pour le thai de Bangkok, diverses tentatives existent (voir Morén et Zsiga 2007). Les cinq tons du thai de Bangkok pourraient par exemple être étiquetés Haut, Bas, Haut+Bas, Bas+Haut, et «ton zéro » (non spécifié, réalisé comme Moyen), se fondant sur une stylisation approximative de leur contour. Mais le linguiste est alors démuni pour aborder le changement évoqué ci-dessus : comment le ton 4, de descendant, a pu devenir montant sans solution de 
continuité. Il paraît clair que ce changement n'affecte pas la nature des unités phonologiques en jeu. L'analyse du thai en tons ponctuels* ne fournit pas d'instruments adéquats pour comprendre l'évolution diachronique, et la diversité dialectale qui en constitue la projection dans l'espace.

\section{CONCLUSION}

En conclusion, il importe de réaffirmer que tons ponctuels* et tons complexes* constituent deux types non réductibles l'un à l'autre. Dans le cas des premiers, il est généralement facile d'établir de façon probante leur nature phonologique, par l'examen d'alternances morpho-phonologiques; dans le cas des seconds, en revanche, il est difficile de démontrer positivement que l'on a affaire à des tons complexes, non décomposables. On peut espérer que les progrès réalisés peu à peu dans la compréhension de l'évolution diachronique de l'un et l'autre type de système tonal permettront, à terme, une modélisation phonologique affinée.

\section{APPENDICE : PRINCIPAUX CONCEPTS EMPLOYES}

Ce court glossaire vise à expliciter l'emploi qui est fait ici de termes qui ont des acceptions très diverses d'un auteur à l'autre.

\section{Panchronique (phonologie panchronique).}

Une loi panchronique est «une loi induite à partir d'un relevé typologique d'événements diachroniques précis dont l'analyse révélera les conditions communes d'apparition » (Mazaudon et Michailovsky 2007). Le programme de la phonologie panchronique est posé par Haudricourt de la façon suivante :

Les lois phonétiques (de la grammaire comparée) établissent les conditions nécessaires et suffisantes d'un changement phonologique (ou phonétique) dans une langue déterminée à une époque déterminée. Je pense qu'il est possible d'obtenir des lois analogues en linguistique générale, valables pour toutes les langues à toutes les époques. (...)

Exemple de loi :

Dans toute langue et à toute époque st- initial de mot devient voyelle + st- lorsque les conditions suivantes sont réalisées : 1 . st- initial n'est pas beaucoup plus fréquent que voyelle + st- (relation à exprimer en chiffres) ; 2 . voyelle + st- existe en fin de mot ; 3. Pas d'accent d'intensité sur la syllabe initiale des mots ; 4. Si le mot où s'opère le changement a $\mathrm{N}$ syllabes, il doit exister dans la langue des mots de $\mathrm{N}+1$ syllabes. (Haudricourt 1940.)

Phonologie panchronique. Voir ci-dessus « Panchronique ». 
Registre; registre de type phonatoire (anglais: register; phonation-type register). Dans les langues à registres, le type phonatoire a un rôle distinctif au plan lexical. Ainsi, la langue mon oppose un registre de voix soufflée à un registre de voix modale, ce qui était encore le cas du khmer à date récente (Henderson 1952). Plus encore que d'autres traits linguistiques, les registres tendent à avoir de multiples corrélats (mode de vibration des plis vocaux, mais aussi longueur plus grande des rimes porteuses d'un type phonatoire non modal, différences dans l'articulation vocalique, différences de fréquence fondamentale) et à connaître une forte variabilité entre locuteurs. Le terme "registre » est préféré ici à celui, trop général, de « qualité de voix » : «In a forthcoming book on general phonetics Professor David Abercrombie of Edinburgh accepts 'register' as an appropriate phonological term but suggests that 'phonation-type' is a more suitable term for its phonetic realization, thus reserving the expression 'voice quality' for more general use. » (Henderson 1965:402, note 3.)

Ton ou tonème (anglais: tone, ou toneme). Le terme tonème est strictement équivalent à ton dans l'usage qui est fait ici de ces termes. Le mot tonème vise à faire ressortir le parallélisme avec phonème: le ton est lui aussi une unité qui s'oppose à d'autres au sein d'un système (définition paradigmatique). En cela, le ton n'est pas de même nature que l'accent lexical, lequel a valeur contrastive et non oppositive : ce qui compte est son placement (définition syntagmatique).

La caractérisation fournie ici du terme de «ton » est fonctionnelle. Au plan phonétique, il parait utile, afin d'ébaucher une limite entre tons* et registres*, de préciser que la hauteur (au plan acoustique : la fréquence fondamentale) tient une place de premier plan dans la réalisation phonétique du ton*, à la différence des registres*, pour lesquels la hauteur a un rôle secondaire. Si l'on souhaite fournir une définition phonétique plus précise, une définition différente devra être fournie pour les tons ponctuels* et pour les tons complexes*. Le corrélat phonétique des premiers est simplement la fréquence fondamentale, d'autres dimensions phonétiques n'intervenant que de façon secondaire ou idiosyncratique (par exemple une phonation murmurée ou laryngalisée pouvant accompagner un ton bas). En revanche, les corrélats phonétiques des tons complexes sont divers, ce qui rend impossible une définition de la notion linguistique de ton par le paramètre phonétique qu'est la fréquence fondamentale (et sa contrepartie perceptive, la hauteur), comme le souligne Rose 1989a.

Ton complexe. Ton* n'étant pas défini par une séquence de tons ponctuels*, mais s'opposant aux autres tons du système concerné par un ensemble de caractéristiques qui peuvent inclure, outre le décours de la hauteur dans le temps (« contour mélodique »), des types de phonation* spécifiques. 
Le terme ton complexe est préféré à celui de ton modulé* (employé par Pike 1948) pour deux raisons. La principale raison est que cette classe inclut les tons d'une langue telle que le vietnamien, dont les tons sont définis non seulement par une certaine modulation de la hauteur au cours de la syllabe, mais aussi par leur type de phonation* (par exemple, la présence d'une constriction glottale en un certain point), tandis que Pike n'envisageait que le paramètre de hauteur: « the glides of a contour system must be treated as unitary tonemes and cannot be broken down into end points which constitute lexically significant contrastive pitches » (Pike 1948:10). D'autre part, le terme ton modulé* est employé dans plusieurs acceptions contradictoires d'un auteur à l'autre (voir l'entrée correspondante dans le présent glossaire), ce qui a incité à préférer un autre terme.

Une même famille de langues peut comprendre aussi bien des langues à tons ponctuels et des langues à tons complexes, comme c'est le cas de la famille sinotibétaine.

Ton modulé (anglais : contour tone). Le terme désigne deux réalités différentes : d'une part, un ton* composé de deux tons ponctuels* (ou plus); d'autre part, un ton complexe* défini par une certaine évolution de la hauteur au cours de la syllabe. Ce terme peut prêter à malentendu : Pike 1948 oppose tons ponctuels* et tons modulés (contour tones), les seconds n'étant pas réductibles aux premiers ; mais cette distinction se heurte à l'habitude bien établie d'employer la même expression de ton modulé pour faire référence à des séquences de tons ponctuels (par exemple, une séquence LH réalisée sur une seule syllabe est appelée « contour »).

Ton ponctuel (anglais : level tone). Ton* défini par un niveau discret de hauteur relative, ou par une séquence de niveaux. Par exemple, dans une langue distinguant deux niveaux, on peut trouver les tons suivants: Haut, Bas, Haut+Bas, et Bas+Haut. Les systèmes de tons ponctuels peuvent distinguer deux à cinq niveaux. Les systèmes à plus de trois niveaux sont peu courants (exemple : le bariba, cf Welmers 1952). Les systèmes à cinq niveaux sont rares (exemple : le bench, ou gimira, cf Wedekind 1983, 1985xxxxx); un seul cas de système recourant à six niveaux a été rapporté (la langue chori : Dihoff 1977, pour laquelle une réanalyse est possible : cf Odden 1995). Cinq ou six peut paraitre un chiffre peu élevé, au regard du nombre de notes musicales que l'oreille peut distinguer; mais dans la parole, où l'attention ne peut évidemment pas se concentrer exclusivement sur la reconnaissance des hauteurs successives, les systèmes de quatre ou cinq tons présentent de fait un degré élevé de complexité. Dans le domaine asiatique, à ma connaissance, le nombre le plus 
élevé de tons ponctuels observés est de trois (exemple: le naxi, cf Michaud 2006).

Type de phonation (anglais : phonation type). Mode de vibration des plis vocaux au cours de la phonation. Ces phénomènes sont souvent appelés qualité de voix (anglais : voice quality), mais Abercrombie recommande de réserver ce dernier terme à un emploi plus général. 


\section{REFERENCES}

Bao Z., 1999, The Structure of Tone, New York/Oxford, Oxford University Press.

Barrie M., 2007, Contour tones and contrast in Chinese languages, Journal of East Asian Linguistics, 16, pp. 337-362.

Brunelle M., 2009, Tone perception in Northern and Southern Vietnamese, Journal of Phonetics, 37, pp. 79-96.

Chao Yuen-ren, 1929, Beiping intonation [in Chinese], in A.A. Milne: The Camberley Triangle, Shanghai, Zhonghua Bookstore, pp. (Appendix).

Chao Yuen-ren, 1933, Tone and intonation in Chinese, Bulletin of the Institute of History and Philology, Academia Sinica, 4:3, pp. 121-134.

Chirkova E. et Michaud A., 2009, Approaching the prosodic system of Shixing, Language and Linguistics, 10(3), pp. 539-568.

Clements N. et Goldsmith J., 1984, Autosegmental studies in Bantu Tone, Publications in African Languages and Linguistics, Dordrecht, Foris.

Clements N., Michaud A. et Patin C., forthcoming, Do we need tone features?, in Tones and Features, E. Hume, J. Goldsmith et W. L. Wetzels.

Dihoff I. R., 1977, Aspects of the tonal structure of Chori, Ph.D., University of Wisconsin.

Ding P. S., 2006, A Typological Study of Tonal Systems of Japanese and Prinmi: Towards a Definition of Pitch-accent Languages, Journal of Universal Language, 7, pp. 1-35.

Evans J., 2008, 'African' tone in the Sinosphere, Language and Linguistics, 9(3), pp. 463-490.

Ferlus M., 1979, Formation des registres et mutations consonantiques dans les langues mon-khmer, Mon-Khmer Studies, 8, pp. 1-76.

Ferlus M., 1998, Les systèmes de tons dans les langues viet-muong, Diachronica, 15(1), pp. 1-27.

Ferlus M., 2001, The Origin of Tones in Viet-Muong, Text of communication at the 11th Annual Conference of the Southeast Asian Linguistic Society XI, Bangkok, Institute of Languages and Culture, Mahidol University (publication of Proceedings is currently under way).

Ferlus M., 2009, What were the four divisions of Middle Chinese?, Diachronica, 26(2), pp. 184-213.

Hagège C. et Haudricourt A.-G., 1978, La phonologie panchronique, Paris, Presses Universitaires de France.

Haudricourt A.-G., 1940, Méthode pour obtenir des lois concrètes en linguistique générale, Bulletin de la Société de Linguistique de Paris, 41(1), pp. 70-74. 
Haudricourt A.-G., 1954, De l'origine des tons en vietnamien, Journal Asiatique, 242, pp. 69-82.

Haudricourt A.-G., 1961, Bipartition et tripartition des systèmes de tons dans quelques langues d'Extrême-Orient, Bulletin de la Société de Linguistique de Paris, 56(1), pp. 163-80.

Haudricourt A.-G., 1969, La linguistique panchronique nécessaire à la linguistique comparée, science auxiliaire de la diachronie sociologique et ethnographique, Ethnies, 3, pp. 23-26.

Henderson E. J. A., 1952, The main features of Cambodian pronunciation, Bulletin of the School of Oriental and African Studies, 14(1), pp. 149-174.

Henderson E. J. A., 1965, The topography of certain phonetic and morphological characteristics of South East Asian languages, Lingua, 15, pp. 400-434.

Hildebrandt K., 2003, Manange tone: scenarios of retention and loss in two communities, $\mathrm{PhD}$, Linguistics, Santa Barbara, U. of California at Santa Barbara. Hyman L. M., 2001, Privative Tone in Bantu, in Proceedings of the symposium "Cross-linguistic studies of tonal phenomena: Tonogenesis, Japanese Accentology, and Other Topics”, S. Kaji, Tokyo, Tokyo University of Foreign Studies, Institute for the Study of Languages and Cultures of Asia and Africa, pp. 237-258.

Hyman L. M., 2006, Word-prosodic typology, Phonology, 23, pp. 225-257.

Hyman L. M., 2007a, How (not) to do phonological typology: the case of pitchaccent, Berkeley, UC Berkeley, UC Berkeley Phonology Lab Annual Report, pp. 654-685.

Hyman L. M., 2007b, Kuki-Thaadow: an African tone system in Southeast Asia, Berkeley, UC Berkeley, UC Berkeley Phonology Lab Annual Report, pp. 1-19.

Hyman L. M. et VanBik K., 2002, Tone and stem2 formation in Hakha Lai, Linguistics of the Tibeto-Burman Area, 25, pp. 113-121.

Mazaudon M., 1978, Consonantal mutation and tonal split in the Tamang subfamily of Tibeto-Burman, Kailash, 6(3), pp. 157-79.

Mazaudon M., 2005, On tone in Tamang and neighbouring languages: synchrony and diachrony, in Proceedings of the symposium "Cross-linguistic studies of tonal phenomena: Tonogenesis, Japanese Accentology, and Other Topics”, S. Kaji, Tokyo, Tokyo University of Foreign Studies, Institute for the Study of Languages and Cultures of Asia and Africa, pp. 79-96.

Mazaudon M. et Michailovsky B., 2007, La phonologie panchronique aujourd'hui: quelques repères, in Combats pour les langues du monde: hommage à Claude Hagège, J. Fernandez-Vest, Paris, L'Harmattan, pp. 351-362.

Michaud A., 2006, Tonal reassociation and rising tonal contours in Naxi, Linguistics of the Tibeto-Burman Area, 29(1), pp. 61-94. 
Michaud A. et He Xueguang, 2007, Reassociated tones and coalescent syllables in Naxi (Tibeto-Burman), Journal of the International Phonetic Association, 37(3), pp. 237-255.

Morén B. et Zsiga E., 2007, The lexical and post-lexical phonology of Thai tones, Natural Language and Linguistic Theory, 24(1), pp. 113-178.

Odden D., 1995, Tone: African languages, in Handbook of Phonological Theory, J. Goldsmith, Oxford, Blackwell, 444-475.

Pike K. L., 1948, Tone Languages. A Technique for Determining the Number and Type of Pitch Contrasts in a Language, with Studies in Tonemic Substitution and Fusion, Ann Arbor, University of Michigan Press.

Pittayaporn P., 2007, Directionality of tone change, International Conference of the Phonetic Sciences, Saarbrücken, pp. 1421-1424.

Pulleyblank E. G., 1978, The nature of the Middle Chinese tones and their development to Early Mandarin, Journal of Chinese Linguistics, 6, pp. 173-203.

Rialland A., 2001, Anticipatory Raising in Downstep Realization: Evidence for Preplanning in Tone Production, in Proceedings of the symposium "Crosslinguistic studies of tonal phenomena: Tonogenesis, Japanese Accentology, and Other Topics", S. Kaji, Tokyo, Tokyo University of Foreign Studies, Institute for the Study of Languages and Cultures of Asia and Africa, pp. 301-322.

Rose P., 1982, Acoustic characteristics of the Shanghai-Zhenhai syllable-types, in Papers in Southeast Asian Linguistics $n^{\circ} 8$ : Tonation, D. Bradley, Canberra, Australian National University Press, Pacific Linguistics, Series A, nº62, pp. 153.

Rose P., 1989a, On the non-equivalence of fundamental frequency and linguistic tone, in Prosodic Analysis and Asian Linguistics: to honour R.K. Sprigg, D. Bradley, E. J. A. Henderson et M. Mazaudon, Canberra, Pacific Linguistics C104, pp. 55-82.

Rose P., 1989b, Phonetics and phonology of Yang tone phonation types in Zhenhai, Cahiers de linguistique - Asie Orientale, 18, pp. 229-245.

Rose P., 1990, Acoustics and phonology of complex tone sandhi: An analysis of disyllabic lexical tone sandhi in the Zhenhai variety of $\mathrm{Wu}$ Chinese, Phonetica, 47, pp. 1-35.

Sagart L., 1999, Tibeto-Burman Tonology in an Areal Context, in Proceedings of the symposium "Cross-linguistic studies of tonal phenomena: Tonogenesis, Japanese Accentology, and Other Topics”, S. Kaji, Tokyo, Tokyo University of Foreign Studies, Institute for the Study of Languages and Cultures of Asia and Africa, pp. 91-104.

Sun J. T.-S., 1997, The Typology of Tone in Tibetan, Chinese Languages and Linguistics IV: Typological studies of languages in China, Taipei, Taiwan, 
Symposium Series of the Institute of History and Philology-Academia Sinica, pp. 485-521.

Svantesson J.-O., 2001, Tonogenesis in Southeast Asia: Mon-Khmer and beyond, in Proceedings of the symposium "Cross-linguistic studies of tonal phenomena: Tonogenesis, Japanese Accentology, and Other Topics”, S. Kaji, Tokyo, Tokyo University of Foreign Studies, Institute for the Study of Languages and Cultures of Asia and Africa, pp. 45-58.

Wan I.-P. et Jaeger J., 1998, Speech errors and the representation of tone in Mandarin Chinese, Phonology, 15, pp. 417-461.

Welmers W. E., 1952, Notes on the structure of Bariba, Language, 28, pp. 82103.

Yip M., 1989, Contour tones, Phonology, 6, pp. 149-174. 